\title{
Endoscopic molecular imaging in inflammatory bowel disease
}

\author{
Nam Seok Ham ${ }^{1}$, Seung-Jae Myung ${ }^{2}$ \\ ${ }^{1}$ Department of Gastroenterology, Veterans Health Service Medical Center, Seoul; ${ }^{2}$ Department of Gastroenterology, Digestive Diseases Research \\ Center, Asan Medical Center, University of Ulsan College of Medicine, Seoul, Korea
}

\begin{abstract}
Molecular imaging is a technique for imaging the processes occurring in a living body at a molecular level in real-time, combining molecular cell biology with advanced imaging technologies using molecular probes and fluorescence. Gastrointestinal endoscopic molecular imaging shows great promise for improving the identification of neoplasms, providing characterization for patient stratification and assessing the response to molecular targeted therapy. In inflammatory bowel disease, endoscopic molecular imaging can be used to assess disease severity and predict therapeutic response and prognosis. Endoscopic molecular imaging is also able to visualize dysplasia in the presence of background inflammation. Several preclinical and clinical trials have evaluated endoscopic molecular imaging; however, this area is just beginning to evolve, and many issues have not been solved yet. In the future, it is expected that endoscopic molecular imaging will be of increasing interest among clinicians as a new technology for the identification and evaluation of colorectal neoplasm and colitis-associated cancer. (Intest Res 2021;19:33-44)
\end{abstract}

Key Words: Molecular imaging; Intestinal diseases; Inflammatory bowel disease; Colitis; Neoplasm

\section{INTRODUCTION}

An endoscope for the diagnosis of digestive diseases was invented 150 years ago by Kussmaul. ${ }^{1}$ Having undergone rapid development, endoscopy is now used for diagnosing and treating many gastrointestinal (GI) diseases, with optical developments allowing very detailed observations. Although current endoscopy is able to achieve very high image quality, it is still on the path to becoming a more complete diagnostic device. White light endoscopy (WLE) plays an important role in early diagnosing of GI lesions, but many studies have reported that the rate of missed lesion detections has not yet decreased. ${ }^{2-4}$ The latest technological and scientific developments

Received December 16, 2019. Revised January 29, 2020.

Accepted January 31, 2020.

Correspondence to Seung-Jae Myung, Department of Gastroenterology, Asan Medical Center, University of Ulsan College of Medicine, 88 Olympic-ro 43-gil, Songpa-gu, Seoul 05505, Korea. Tel: +82-2-3010-3917, Fax: +82-2-

476-0824, E-mail: sjmyung@amc.seoul.kr of GI endoscopy have created new imaging devices, such as virtual chromoendoscopy techniques, chromoendoscopy, narrow-band imaging, autofluorescence imaging, Raman spectroscopy and confocal laser endomicroscopy (CLE), all of which can help to improve the characterization and detection of early neoplasms. ${ }^{5}$ Although these advances, endoscopy is diagnostically limited because it discriminates based on morphological changes. Recently, molecular mechanisms at the cellular level have been revealed for many physiological and pathological conditions such as cancer and inflammation. Therefore, to significantly increase the early detection rate of endoscopic examination, a method of searching for changes at the cellular level, rather than for morphological changes, has been devised.

Molecular imaging is a technique for imaging the processes occurring in a living body at the molecular level, combining advanced imaging technology with molecular cell biology. Molecular imaging has evolved, and it is now able to reveal the molecular pathogenesis of many diseases. It has developed 
particularly rapidly in the field of nuclear medicine, where it is used for the clinical diagnosis of many important diseases such as cancers, dementia, and Parkinson disease. Much research has been expended to apply molecular imaging to the field of endoscopy, with studies describing applications in cancer screening and surveillance, as well as its use for the evaluation of pathological conditions such as gastric intestinal metaplasia, Barrett esophagus, colitis-associated cancer (CAC), flat and depressed sporadic colonic adenomas, and indeterminate biliary strictures. ${ }^{6}$

The principles of endoscopic molecular imaging and its applications in intestinal diseases have been summarized in previous reviews. ${ }^{7,8}$ The uses of molecular imaging in intestinal diseases include increasing the diagnosis rate of precancerous lesions, confirming the characteristics of the lesions, and confirming the response to treatment with anticancer drugs. In this review, we summarize the use of molecular imaging for the early detection and evaluation of colonic neoplasia and CAC in inflammatory bowel disease (IBD).

\section{DEVICES AND PROBES FOR ENDOSCOPIC MO- LECULAR IMAGING}

When using disease-specific biomarkers as molecular probes, it is important to consider several aspects in selecting them, including rapid binding kinetics, the ease of labeling with fluorophores, target specificity, low immunogenicity and deep tissue penetration. Recent studies used fluorescence-labeled exogenous molecular probes such as peptides, antibodies, activatable probes, and nanoparticles. ${ }^{6}$

For molecular imaging in the GI tract, the imaging device should be able to detect the molecular probes at a concentration that binds to the region of interest in a minimally invasive manner and that is enough to distinguish molecular changes in the early stage of neoplasms. Using highly sensitive widefield endoscopes is integral part if cellular details are to be detected during a procedure. Recently, customized or experimental endoscopes have been developed for the detection and characterization of GI lesions using molecular imaging techniques such as fluorescence endomicroscopy, CLE, and optical coherence tomography. ${ }^{5}$

CLE shows enormous potential to replace biopsy and histological diagnosis. That is, we can proceed to the next therapeutic stage of clinical practice without waiting for pathologic results. ${ }^{9-11}$ However, most clinical trials used only fluorescein for fluorescent staining in morphological distinction. ${ }^{12}$ The U.S.
Food and Drug Administration approved the use of intravenous fluorescein for ophthalmological imaging of blood vessels, and this method therefore offers the advantage of ensuring safety but it only allows better morphological distinction; it does not target tumors directly, and new fluorescent probes need to be developed in the future.

Photoacoustic tomography (PAT) is the most advanced form of research in the field of molecular imaging. By acoustically detecting optical absorption contrast, Yao and Wang ${ }^{13}$ reported that PAT broke the penetration limits of traditional high-resolution optical imaging and conventional molecular imaging. Through spectroscopic analysis of the target's optical absorption, PAT can identify a wealth of endogenous and exogenous molecules and is therefore inherently capable of molecular imaging with high sensitivity. The molecular sensitivity of PAT is accompanied by the advantages of no ionizing radiation, high spatial resolution, and deep penetration of biological tissues, which other optical imaging modalities cannot yet achieve. Yang et al. ${ }^{14}$ also demonstrated simultaneous functional photoacoustic and ultrasonic dual-mode endoscopy, and showed its ability to image internal organs in vivo. Photoacoustic endoscopy is ideal for soft tissue imaging because it has high optical contrast and spatial resolution, even in relatively deep tissues.

Because of these advantages, photoacoustic endoscopy can surmount the limitations of ultrasound endoscopy. Moreover, the benefits of photoacoustic imaging do not sacrifice the advantages of existing ultrasound functions; photoacoustic endoscopy systems are inherently compatible with ultrasound imaging, enabling multimodality imaging with complementary contrast.

\section{EARLY DETECTION OF COLONIC NEOPLASIA USING MOLECULAR IMAGING}

The adenoma missing rate remains about $22 \%(15 \%-32 \%),{ }^{3}$ with depressed and flat adenoma being frequently missed. Molecular imaging can improve the detection rate of missed adenoma including flat/depressed lesions, and residual adenoma after endoscopic resection.

In a study using adenomatous polyposis coli (APC) genemutated mice, a cathepsin-sensing near-infrared probe was used for fiberoptic endoscopic detection of colorectal adenomas, ${ }^{15}$ demonstrating that tumor-specific dequenching of smart probes can be used for endoscopic imaging. In a recent study, Miller et al. ${ }^{16}$ and Joshi et al. ${ }^{17}$ used an in vivo phage dis- 
play to recognize peptides that bind adenoma in a colorectal tumorigenesis mouse model. Morphologic and molecular studies have shown that the histopathologic manifestations of colorectal tumors in the APC mouse model mimic those of human colorectal tumors. Fluorescently labeled peptides bound dysplastic epithelium in mouse model. APC mice were monitored through a wide-field endoscope. Multispectral peptides can be used to obtain colorful images of tumor's molecular profiles. ${ }^{18-20}$

Near-infrared fluorescence (NIRF) imaging was carried out by Yoon et al. ${ }^{21,22}$ using a matrix metalloproteinase (MMP)-activatable probe to discover colorectal neoplasm at an early stage of disease. NIRF signal intensities correlated closely with each stage of disease from adenoma to adenocarcinoma. In addition, NIRF imaging allowed differentiation of inflammatory mucosa from tumors. This strategy made it possible to evaluate therapeutic effects earlier, before morphological changes. NIRF imaging combined with a polymeric nanoparticle-based probe improves detection rate of tumor and allows estimation of the possibility of malignant change of the tumor.

Burggraaf et al. ${ }^{23}$ developed an intravenously administrable fluorescent probe specific for a polyp biomarker that is safe and well tolerated in humans. GE-137 is a water-soluble probe consisting of a 26-amino acid cyclic peptide conjugated to a fluorescent cyanine dye that binds to human tyrosine kinase (c-Met). Intravenous administration of GE-137 leads to its specific accumulation in c-Met-expressing tumors in mice. In patients receiving GE-137 intravenously, fluorescence colonoscopy enabled visualization of all neoplastic polyps that could be seen with white light, as well as additional polyps that could not be seen with white light. This first-in-human pilot study shows that molecular imaging with an intravenous fluorescent agent is safe and feasible and can detect polyps not detectable by WLE (Fig. 1).

During GI endoscopy, fluorescence probes for molecular imaging can be applied topically and even systemically, although it is more difficult to ensure the safety of intravenously administered probes. The safety of probes should be tested extensively through in vitro genotoxicity and phototoxicity studies. Biodistribution, pharmacology, and general toxicity studies can only be performed in humans after no adverse event has been observed in studies conducted on rats and monkeys. After drug administration, endoscopic fluorescence measurements of healthy volunteers should be performed, including repeated examinations to estimate the optimal dose, optimal time interval, or optimal imaging time point between drug ad- ministration and endoscopy. In addition, blood levels and clearance rates after dosing should also be considered.

Because of these difficulties, many spraying-type probes have been developed, and their effectiveness has been demonstrated in the laboratory. Park et al. ${ }^{24}$ developed antibodyquantum dots (Ab-QDs) that were sprayed and washed simultaneously onto colon tumor tissues inside live animals, and the tissues were then subsequently excited and imaged by endoscopy. The QDs were conjugated to various Abs, such as MMP9, MMP14, and carcinoembryonic antigen. Ab-QD probes can diagnose tumors in the mouse colon ex vivo and in vivo, as well as in human tissues. The imaging can be completed in 30 minutes, and tissue can be imaged to a depth of $100 \mu \mathrm{m}$. The probes successfully detected areas of hyperplasia and adenoma, as well as tumors clearly are visible to the human eye.

In addition, antibody-type probes, such as the indocyaninebased fluorescent probe that is readily activated by the tumorenriched enzyme $\gamma$-glutamyltranspeptidase $(\gamma \mathrm{GT}){ }^{25}$ anti-CCSP (colon cancer secreted protein) -2 antibody, ${ }^{26}$ and PSP1 (phosphatidylserine-recognizing peptide 1) as an apoptosistargeting probe, ${ }^{27}$ have been developed, and their usefulness has been demonstrated in the laboratory. The advantage of these ex vivo experiments is that they are relatively free from safety issues in comparison to intravenous administration. However, spraying-type probes can only be applied where direct access to the subject is possible and the area of interest is restricted. In addition, in vivo studies are still limited because of safety concerns, and these probes have only currently been tested in small animal models. Spray-type probes also require long staining times and offer only shallow tissue penetration.

Recently, Park et al. ${ }^{24}$ reported a study that made up for the QD-Ab shortcomings of marginal specificity and a long timerequirement. They demonstrated a ratiometric fluorescent probe, crystal violet (CV)-glutamic acid (Glu), which responds to $\gamma \mathrm{GT}$ activity, and was developed and co-used with an MMP14 Ab-biocompatible AgInS2/ZnS QD conjugate probe. This work demonstrated the potential for endoscopic early colon cancer diagnosis by the multiplexed and complementary detection of colorectal tumors and precancerous regions such as adenoma and hyperplasia. ${ }^{28}$ The CV-Glu probe clearly indicated tumor sites within 5 minutes on fresh mouse colon tissues and human colon adenoma specimens. The co-use of CV-Glu and MMP14-Ab-QD probes further enhanced diagnostic power, as the probe signals showed different spatial distributions, thereby facilitating exploitation of complemen- 

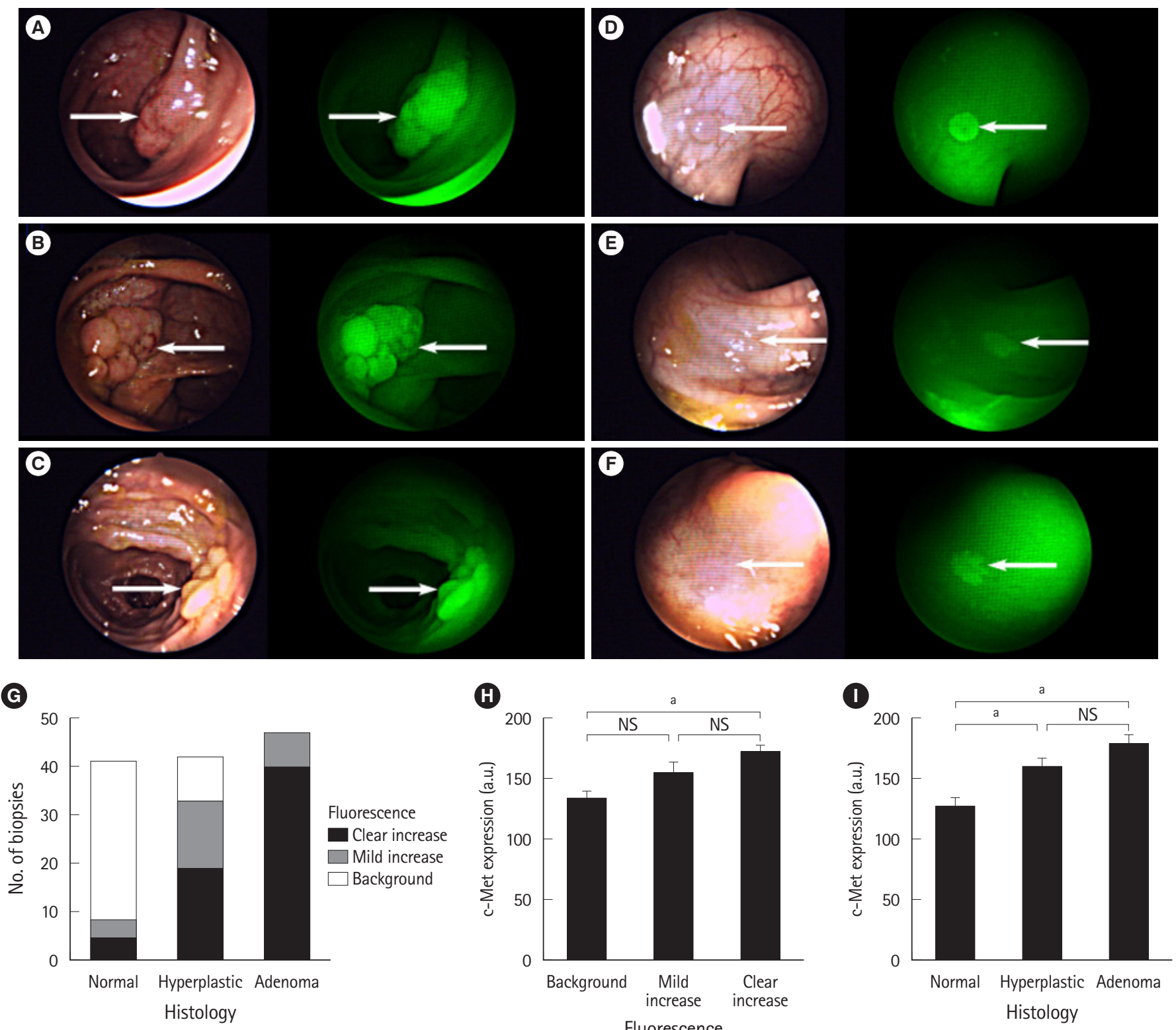

Fig. 1. Simultaneous white light (WL) and fluorescent light $(\mathrm{FL})$ images of representative lesions of various morphological and histological subtypes found. (A-C) The lesions shown are clearly visible in WL and show clearly increased fluorescence. (D) A lesion that, although it was visible in WL, had enhanced visibility in FL. (E, F) Images representative of the 9 lesions that were only visible in FL. Polyps are indicated by the white arrows. (A) A 2-cm pedunculated (Paris 0-Ip) tubular adenoma. (B) A 4-cm subpedunculated (Paris 0-Isp) tubulovillous adenoma. (C) A 2-cm sessile (Paris 0-Is) serrated polyp. (D) A 5-mm flat elevated (Paris 0-Ila) tubular adenoma. (E, F) Flat (Paris 0-IIb) tubular adenomas, $5 \mathrm{~mm}$ and $4 \mathrm{~mm}$ in diameter, respectively. (G) Graph showing the relationship between the degree of fluorescence and the histological diagnosis. (H) Graph showing the relationship between c-Met expression and the degree of fluorescence. (I) Graph showing the relationship between c-Met expression and histological diagnosis. Error bars are means \pm standard error of the mean. ${ }^{a} P<0.001$, mixed-model analysis of variance. Adapted from Burggraaf J, et al. Nat Med 2015;21:955-961, with permission from Springer Nature. ${ }^{23}$

tary information (Fig. 2).

These various devices and probes provide a significant advantage for the detection of the small or flat tumors that may be missed on conventional endoscopic examinations, and they provide a strategy for the improvement of cancer diagnosis.

\section{EARLY DETECTION OF COLITIS-ASSOCIATED CANCER IN IBD}

\section{Current Strategies for Detection of Neoplasm in IBD}

Fig. 3 shows that the techniques and equipment used for en- 


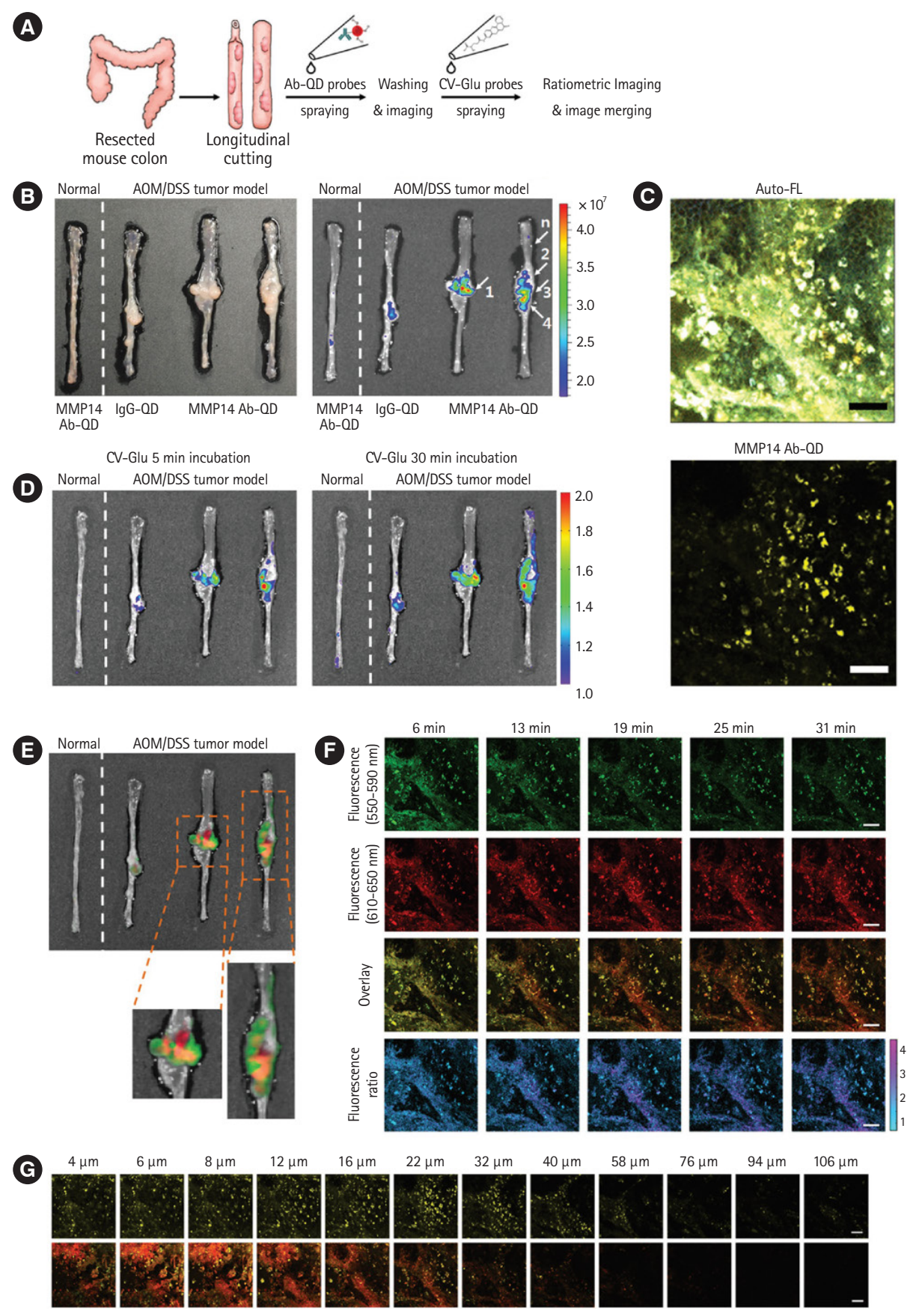

Fig. 2. (A) Schematic diagram of sequential ex vivo staining with crystal violet (CV)-glutamic acid (Glu) and matrix metalloproteinases 14 (MMP14) antibody-quantum dot (Ab-OD) probes. (B) White light images (left panel) and in vivo imaging system images (right panel) of (from left) a normal colon tissue treated with the MMP14 Ab-OD probe, a tumor tissue treated with IgG-QD conjugates, and two other tumor colons treated with the MMP14 Ab-OD probe. Five regions were histopathologically analyzed (arrows 1, 2, 3, 4, and n). (C) Twophoton microscopy (TPM) images of mouse tumor colon stained by the MMP14 Ab-QD probe. Upper image: autofluorescence (Auto-FL) imaging, lower image: MMP14 Ab-QD probe signals (yellow pseudo-color). (D) Ratiometric signals after spraying all tissues with the CVGlu probe at 5 and 30 minutes after treatment. (E) Overlay images of the CV-Glu probe radio frequency signal at 30 minutes (green pseudo-color) and MMP14 Ab-OD probe signal (red pseudo-color). (F) Time-dependent TPM images of the CV-Glu probe in the same area

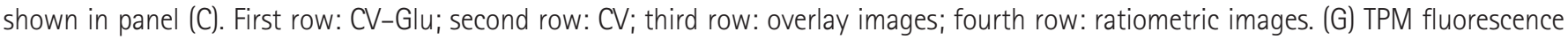
images recorded moving down in the z-direction. First row: MMP14 Ab-QDs (yellow pseudo-color); second row: overlay images of CV-Glu probe. Scale bar: 50 m. AOM, azoxymethane; DSS, dextran sodium sulfate. Adapted from Park Y, et al. Acs Nano 2014;8:8896-8910, with permission from John Wiley and Sons. ${ }^{24}$ 

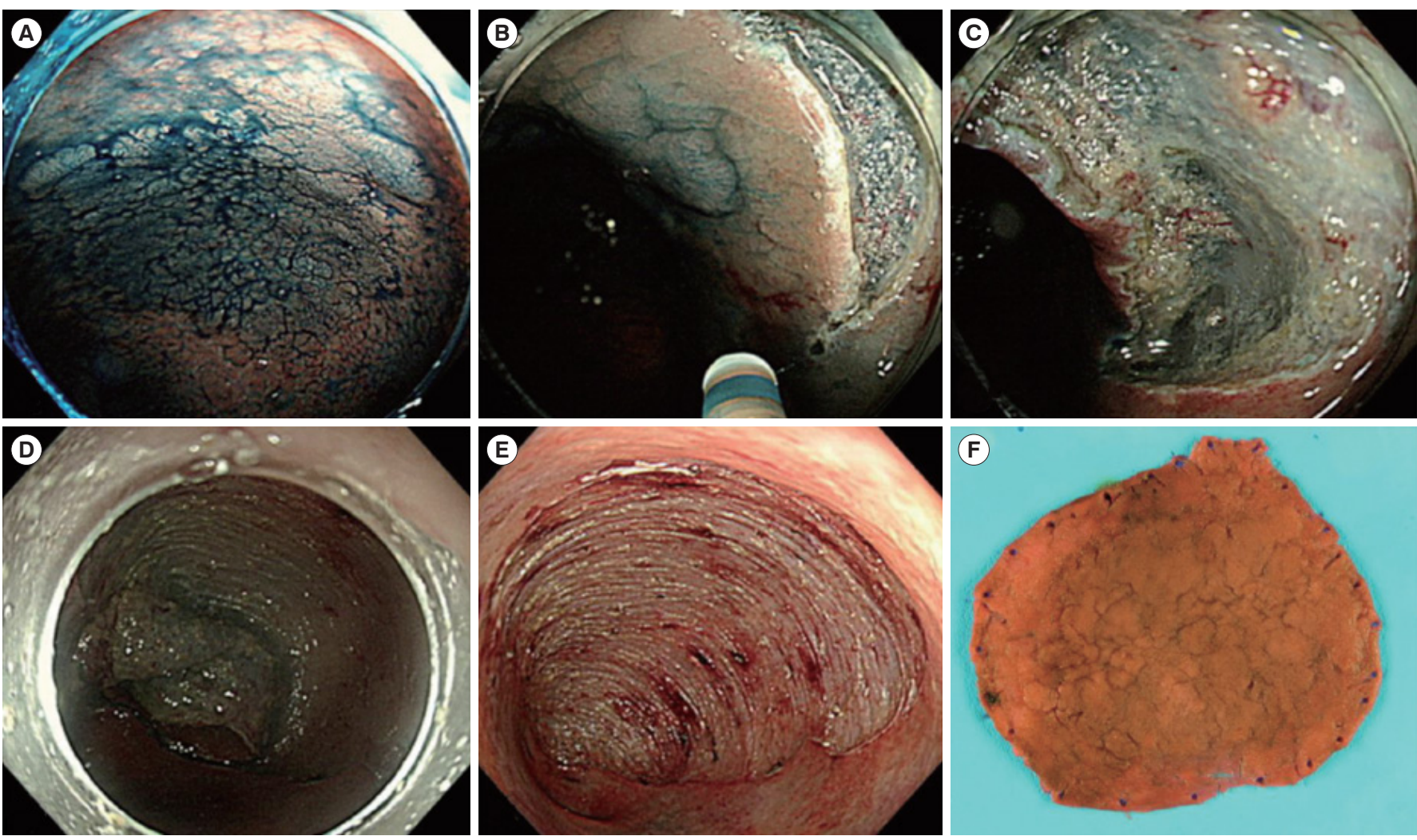

Fig. 3. Representative example of endoscopic submucosal dissection for colitis-associated dysplasia. (A) Large, non-ulcerated Paris type 0 -lla dysplasia with a distinct border in the rectum. (B) Mucosal incision was performed after submucosal injection. (C) Mild but diffuse submucosal fibrosis and submucosal fat deposition. (D-F) The colonoscope was changed into a gastroscope to expose the submucosal layer more effectively, and en bloc resection was achieved. The final histology revealed low-grade dysplasia $(42 \times 40 \mathrm{~mm}$ in size, with clear lateral and vertical margins). Adapted from Yang DH, et al. Clin Endosc 2019;52:120-128. ${ }^{29}$

doscopic submucosal dissection (ESD) for sporadic colorectal neoplasia is also applicable to ulcerative colitis (UC)-associated dysplasia. ${ }^{29}$ To make this possible, the endoscopic specialist must be highly trained in colorectal ESD, taking into account the lesions and the current condition of the IBD patients. In this section, we introduce and summarize the molecular pathobiology of CAC, the current guidelines, and the latest international consensus statement for detection and management of CAC.

Colorectal cancer is a serious complication of long-standing IBD, as demonstrated by meta-analyses of population-based cohort studies, ${ }^{30,31}$ and the incidence of cancer in IBD patients is raising in Korea. ${ }^{32}$ The risk of colorectal cancer in patients with IBD is six times higher than that in the general population and accounts for $10 \%-15 \%$ of deaths in patients with IBD. ${ }^{33}$ Unlike sporadic colorectal cancer, CAC is preceded by clinically detectable IBD, such as Crohn's disease (CD) or UC. ${ }^{34-36}$ The importance of screening and follow-up monitoring through colonoscopy is emphasized for the early diagnosis and treatment of CAC. However, CAC typically occurs at a younger age than sporadic colorectal cancer (40s-50s vs. 60s), and shows a high incidence of multiple-concurrent colon cancer. Furthermore, early detection is difficult because of the flat form and the inflammation of the surrounding mucosa.

Carcinogenic process in the inflamed colon looks different from the sequence of genetic alterations observed in sporadic colorectal cancers. ${ }^{34}$ A study of CAC showed increased levels of oxidative injury, which occurs especially in cancer sites. Chronic colonic inflammation can lead to oxidative DNA damage, which can cause cancer development when it involves key gene of growth regulation. ${ }^{37}$

The molecular pathobiology of CAC has a lot of the identical abnormalities in the factors of cell cycle regulation such as APC, p53, Ki-ras, and the mismatch repair genes (e.g., hMSH2), as those found in sporadic colorectal cancer. ${ }^{38,39}$ Despite these similarities, distinctive features of CAC arise due to differences 
in when and how often these mutation occur. ${ }^{40,41}$

Among major colorectal cancer markers, APC and p53 show critical dissimilarity in when function loss occurs between sporadic colorectal cancer and CAC. In sporadic colorectal cancer, the development of a nonfunctional APC gene, which usually happens before early adenoma, occurs much later in CAC just before the development of carcinoma.

p53 plays an important role in regulating the cell cycle. Lossof-function mutations in this gene happen the identical mechanism and with a similar frequency in both CAC and sporadic colorectal cancer. However, the p53 mutations generally take place late in sporadic colorectal cancer, whereas they develop much earlier in CAC. ${ }^{33}$ In addition, p53 mutations are often discovered in the normal mucosa of IBD patients. This difference in pathogenesis results in morphological differences between the 2 neoplastic forms. ${ }^{40}$

The aim of colonoscopy for colorectal cancer screening is to find premalignant lesions early to allow resection to avoid the problems of invasive cancer. ${ }^{33}$ Dysplasia in IBD can be found at sites distant from the cancer itself, or before the cancer develops, and it is difficult to recognize on colonoscopy because it often arises from flat normal-appearing mucosa. ${ }^{42}$

Current guidelines recommended that patients with IBD undergo regular surveillance colonoscopy for the early detection of dysplasia. ${ }^{43,44}$ Both American and British Gastrointestinal guidelines recommend a screening colonoscopy in all patients with IBD 8-10 years after diagnosis. ${ }^{45-47}$

Traditional surveillance strategy recommends random biopsy throughout the colon. This is because neoplasms in IBD patients have flat and faint feature that may elude detection and progress quickly into advanced cancer. ${ }^{43,48}$ Over the past 30 years, the recommendation has been to perform at least 33 randomized biopsies in all 4 quadrants every $10 \mathrm{~cm}$ along the colon for colitis-related cancer screening. However, such a random biopsy can only sample about $0.03 \%$ of the whole mucosal surface, and there are problems such as the low detection rate of less than 2 per 1,000 biopsies and the excessively high cost. The limited utility of this strategy dictates the need for a more reliable approach to this important problem.

Newer endoscopic technologies, including high-definition endoscopy ${ }^{49}$ and chromoendoscopy, ${ }^{50,51}$ have significantly improved the detection and characterization of neoplastic lesions, and have the potential to alter the surveillance paradigm in IBD in favor of targeted neoplasia detection with endoscopic resection of even advanced precancerous lesions. ${ }^{43}$

With the development of devices such as high-resolution endoscopy, the experience that endoscopy doctors have with flat adenomas and dysplasia in IBD has accumulated. Watanabe et al. ${ }^{52}$ conducted a randomized controlled trial to compare rates of neoplasia detection between targeted and random biopsies in patients with UC. They found that targeted and random biopsies detected similar proportions of neoplasia, and that targeted biopsy appears to be more cost-effective. Accordingly, target biopsy using chromoendoscopy has been recognized as a standard test method. ${ }^{46}$ On the basis of these findings, the U.K. guideline recommends targeted biopsy with chromoendoscopy.

Indeed, investigators are now reporting that most dysplasia discovered in patients with IBD is visible. ${ }^{53,54}$ This observation will likely have a significant impact on the surveillance of dysplasia.

The recent international consensus statement on the Surveillance for Colorectal Endoscopic Neoplasia Detection and Management in Inflammatory Bowel Disease Patients (SCENIC) proposed a new classification system for dysplasia which was identified during surveillance colonoscopy. ${ }^{55,56}$ First, dysplasia should be divided by visibility, with flat dysplasia being classified as visible dysplasia. Second, other inaccurate terms, including DALM (dysplasia-associated lesion or mass), nonadenoma-like dysplasia, and adenoma lesion or mass were no longer used. The Paris classification for sporadic colorectal neoplasia has been adopted to report visible dysplasia. Third, ulcerations and the distinctness of borders are proposed as aspect of endoscopically unresectable dysplasia. ${ }^{55}$ This new classification will be used as more uniform communication tool among endoscopists comparing with previous descriptions for dysplasia.

Total proctocolectomy has been considered as the standard treatment for UC-associated dysplasia. ${ }^{57}$ This is because there are high prevalence of synchronous or metachronous cancer, as well as the technical limitations in confident identification and complete resection of flat dysplastic lesions. However, recent studies have supported the therapeutic role of endoscopic resection for flat dysplasia in IBD patients, and SCENIC have suggested endoscopic resection as a key modality for the treatment of visible and resectable dysplasia in such patients. ${ }^{5,56,58}$

Several studies have shown that ESD can be used to treat colitis-associated dysplasia. ESD can increase the rate of en bloc and curative resection rates, and reduce the risk of complications. ${ }^{59-62}$ Nevertheless, such studies were carried out only in specialized centers. Furthermore, the rates of recurrence were relatively high, and data of long-term outcome are still 

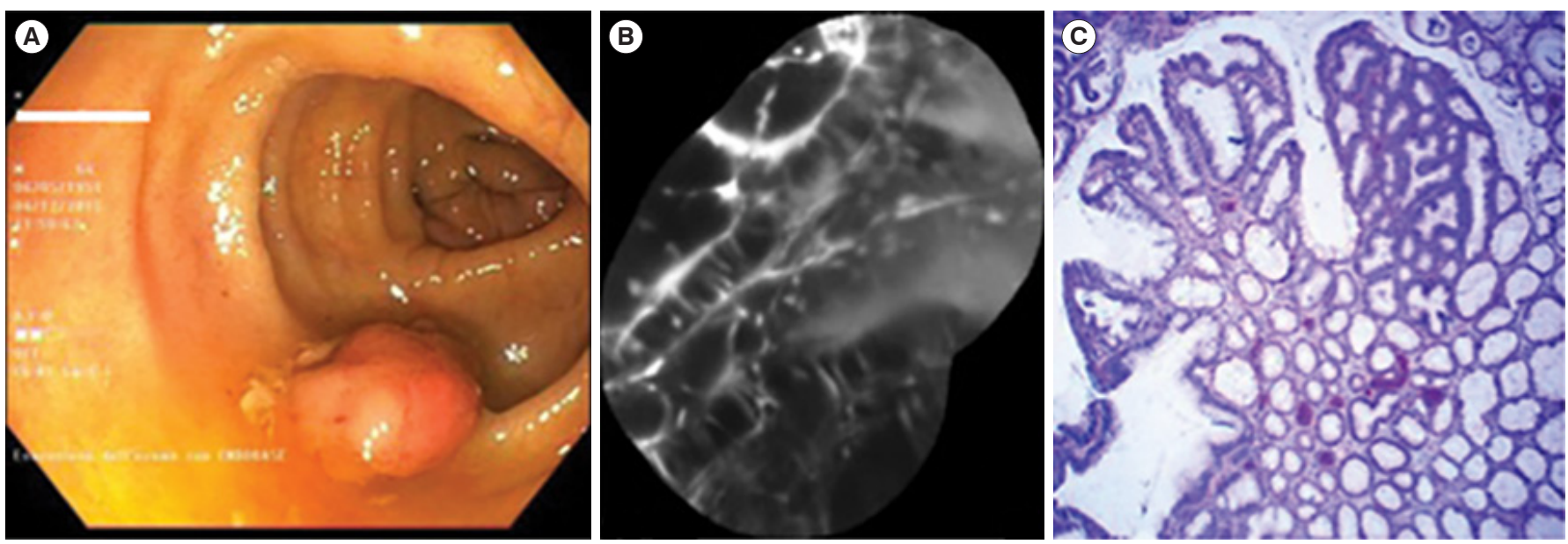

Fig. 4. Dysplastic lesion. (A) White light endoscopic view showing a polypoid lesion (Paris 0-Is) of the transverse colon. (B) After resection and coloration with the $100 \mu \mathrm{M}$ VRPMPLQ peptide solution, confocal laser endomicroscopy shows active binding of the peptide to dysplastic colonocytes is observed. This along with passive accumulation of the peptide determines an increase in fluorescence. (C) Conventional histology $\left(H \& E_{1} \times 106\right)$ showing low-grade dysplasia. VRPMPLO is a synthetic peptide conjugated with fluorescein, which shows more selective binding to dysplastic tissue than to normal mucosa. Adapted from De Palma GD, et al. PLoS One 2017;12:e0180509. ${ }^{67}$

lacking. Therefore, after careful discussion with patients in cooperation with a multidisciplinary IBD team including physicians, surgeons, and pathologists, ESD should be performed for colitis-associated dysplasia.

In summary, the newer endoscopic imaging modalities using molecular imaging are needed to improve the detection and characterization of dysplastic neoplasms in IBD patients, allowing precise targeted access to detection of neoplasm without interval random biopsies, ${ }^{43}$ and enabling endoscopic en bloc and curative resection rather than proctocolectomy.

\section{Identification of Dysplasia in IBD Using Endoscopic Molecular Imaging}

As described above, despite the development of new devices, techniques, and guidelines, acceptance in the real world has been very slow, and techniques, such as those used for the detection and treatment of dysplasia in IBD patients, can only be implemented in a small number of centers. Because of these difficulties, new modalities such as molecular imaging are needed.

Gounaris et al. ${ }^{63}$ reported that cathepsin substrate-based probe imaging precisely recognizes dysplastic lesions within chronically inflamed colons. Cathepsins are lysosomal cysteine proteases that contribute to the proteolytic network in tumor microenvironments. ${ }^{64}$ Gounaris et al. ${ }^{63}$ reported that cathepsin activity of dysplastic tissue could be detected using an endoscope modified to image simultaneously white light and light in the near-infrared spectrum in the colons of colitis-in- duced mice after cathepsin hydrolyzed substrate-based probe injection. Considering that NIRF emission in the local area of dysplasia is much brighter than in the surrounding area of colitis, Gounaris et al. suggested that detection of cathepsin activity allows discrimination of dysplastic tissue from benign areas of active colitis in human biopsies.

Mitsunaga et al. ${ }^{65}$ suggested that topical application of an enzymatically rapid-activatable probe ( $\gamma$-glutamyl hydroxymethyl rhodamine green, gGlu-HMRG) can improve endoscopic detection of CAC. $\gamma \mathrm{GT}$, a cell surface enzyme of glutathione metabolism, is expressed at a higher level in colon cancer cells than in normal colon cells in human ${ }^{66}$ gGlu-HMRG becomes fluorescent after cleavage of a $\gamma$ GT-specific sequence, Mitsunaga et al. showed that gGlu-HMRG fluorescence colonoscopy can be used to image a high cancer to background ratio in a murine colitis-associated colon cancers model. All tissues imaged using this technique included cancer and/or low-to-high grade dysplasia. This cancer-specific, topically sprayed fluorescence probe in conjunction with guided endoscopy will allow the screening of CACs in patients with longstanding IBD who have to undergo repeated screening colonoscopies.

De Palma et al. ${ }^{67}$ reported that ex vivo staining of UC-associated dysplasia using a fluorescent labeled molecular probe and CLE is feasible. This technology, in combination with WLE and chromoendoscopy, can be used to more precisely predict lesion histology and help decision making for lesion 
resectability ${ }^{68,69}$ In this study, a phage-derived heptapeptide with predicted high binding affinity for dysplastic tissue was synthesized and labeled with fluorescein. Dysplasia was associated with active binding of the peptide to dysplastic colonocytes (Fig. 4). Despite these advantages, CLE is limited to expert centers because of its high device price and significant training requirements.

\section{Evaluation of Disease Activity and Prediction of Therapeutic Response in IBD Using Endoscopic Molecular Imaging}

Despite the fact that endoscopy and barium radiological examinations are the gold standards of IBD diagnosis, both techniques require particular preparations, and they are not always tolerated or practicable. Molecular imaging with singlephoton emission tomography ${ }^{70}$ and positron emission tomography (PET) ${ }^{71}$ seem to be trustworthy, noninvasive, precise, and easily reproducible tools which are able to evaluate the location, extent, and activity grade of IBD. However, prospective multicenter studies on larger patient samples are needed for validation. ${ }^{72}$

Wang et al. ${ }^{73}$ reported that ultrasonography with clinically translatable dual-targeted (P- and E-selectin) contrast microbubbles specifically enables detection and quantification of inflammation in a murine acute colitis model, leveraging the natural pathway of leukocyte recruitment in inflammatory tissue. They also demonstrated that ultrasonography imaging with contrast shows a correlation well with fluorodeoxyglucose uptake on PET/computed tomography imaging.

Applying the concept that defects in intestinal barrier function is related with IBD, increased intestinal permeability is also a prognostic indicator of IBD relapse. Kiesslich et al. ${ }^{74}$ reported that cell shedding and barrier loss identified by CLE can predict IBD recurrence and be used as a tool for IBD management.

Atreya et al. ${ }^{75}$ reported that molecular imaging with fluorescent antibodies has the possibility to forecast therapeutic responses to biological treatment and can be used for personalized therapy in CD and inflammatory or autoimmune disorders. Based on this, it is possible to predict treatment algorithms through molecular imaging in IBD before biologic treatment.

\section{CONCLUSIONS}

Molecular imaging endoscopy is expected to develop into a very important test for early detection of GI neoplasms, especially flat and depressed adenoma, and dysplasia in IBD patients. It will also be important for the monitoring of therapy, prediction of therapeutic responsiveness, and determination of treatment methods. An automated precision diagnostic platform will also be developed to help with this. This area is currently in its infancy and many problems remain to be solved. There are potential problems associated with using this probe for molecular imaging, such as poor penetration of the mucus covering the intestinal mucosa, weak binding to specific tissue, and the long time required to acquire an image. In addition, confirming the safety of new markers and conducting a clinical trial in patients are as challenging as obtaining approval of a new drug.

There are many bio-researchers and entrepreneurs developing new technologies, and it is important that clinicians who understand the disease work together to develop new technologies. In the future, many digestive clinicians and researchers will be interested in new technologies such as molecular imaging, with the hope that these new technologies can be used in clinical practice.

\section{ADDITIONAL INFORMATION}

\section{Funding Source}

This research was supported by grants of the Ministry of Trade, Industry \& Energy (MOTIE, Korea) under Industrial Technology Innovation Program (No. 10063408) and the grants from Asan Institute for Life Sciences (Nos. 2019-559 and 2019-261).

\section{Conflict of Interest}

Myung SJ is an editorial board member of the journal but did not involve in the peer reviewer selection, evaluation, or decision process of this article. He is CEO of EdisBiotech Co. No other potential conflicts of interest relevant to this article were reported.

\section{Author Contribution}

Conceptualization: Ham NS, Myung SJ. Writing - original draft: Ham NS, Myung SJ. Writing - review and editing: Myung SJ. Approval of final manuscript: all authors.

\section{ORCID}

Ham NS

Myung SJ

https://orcid.org/0000-0002-6661-4692

https://orcid.org/0000-0003-0585-4016 


\section{REFERENCES}

1. Drossman DA. Challenges in the physician-patient relationship: feeling “drained". Gastroenterology 2001;121:1037-1038.

2. Matloff JL, Abidi W, Richards-Kortum R, Sauk J, Anandasabapathy S. High-resolution and optical molecular imaging for the early detection of colonic neoplasia. Gastrointest Endosc 2011;73:1263-1273.

3. van Rijn JC, Reitsma JB, Stoker J, Bossuyt PM, van Deventer SJ, Dekker E. Polyp miss rate determined by tandem colonoscopy: a systematic review. Am J Gastroenterol 2006;101: 343-350.

4. Yalamarthi S, Witherspoon P, McCole D, Auld CD. Missed diagnoses in patients with upper gastrointestinal cancers. Endoscopy 2004;36:874-879.

5. Dacosta RS, Wilson BC, Marcon NE. New optical technologies for earlier endoscopic diagnosis of premalignant gastrointestinal lesions. J Gastroenterol Hepatol 2002;17 Suppl:S85S104.

6. Goetz M, Wang TD. Molecular imaging in gastrointestinal endoscopy. Gastroenterology 2010;138:828-833.

7. Kim JW, Yoon SM, Myung SJ. Molecular imaging of gastrointestinal malignancies. Intest Res 201 1;9:90-96.

8. Kim SY, Myung SJ. Optical molecular imaging for diagnosing intestinal diseases. Clin Endosc 2013;46:620-626.

9. Shahid MW, Buchner AM, Coron E, et al. Diagnostic accuracy of probe-based confocal laser endomicroscopy in detecting residual colorectal neoplasia after EMR: a prospective study. Gastrointest Endosc 2012;75:525-533.

10. Shahid MW, Buchner AM, Heckman MG, et al. Diagnostic accuracy of probe-based confocal laser endomicroscopy and narrow band imaging for small colorectal polyps: a feasibility study. Am J Gastroenterol 2012;107:231-239.

11. Shahid MW, Buchner AM, Raimondo M, Woodward TA, Krishna M, Wallace MB. Accuracy of real-time vs. blinded offline diagnosis of neoplastic colorectal polyps using probebased confocal laser endomicroscopy: a pilot study. Endoscopy 2012;44:343-348.

12. Wallace MB, Meining A, Canto MI, et al. The safety of intravenous fluorescein for confocal laser endomicroscopy in the gastrointestinal tract. Aliment Pharmacol Ther 2010;31:548552.

13. Yao J, Wang LV. Recent progress in photoacoustic molecular imaging. Curr Opin Chem Biol 2018;45:104-112.

14. Yang JM, Favazza C, Chen R, et al. Simultaneous functional photoacoustic and ultrasonic endoscopy of internal organs in vivo. Nat Med 2012;18:1297-1302.

15. Marten K, Bremer C, Khazaie K, et al. Detection of dysplastic intestinal adenomas using enzyme-sensing molecular beacons in mice. Gastroenterology 2002;122:406-414.

16. Miller SJ, Joshi BP, Feng Y, Gaustad A, Fearon ER, Wang TD. In vivo fluorescence-based endoscopic detection of colon dysplasia in the mouse using a novel peptide probe. PLoS One 2011;6:e17384.

17. Joshi BP, Liu Z, Elahi SF, Appelman HD, Wang TD. Near-infrared-labeled peptide multimer functions as phage mimic for high affinity, specific targeting of colonic adenomas in vivo (with videos). Gastrointest Endosc 2012;76:1197-1206.

18. Zhou L, El-Deiry WS. Multispectral fluorescence imaging. J Nucl Med 2009;50:1563-1566.

19. Miller SJ, Lee CM, Joshi BP, Gaustad A, Seibel EJ, Wang TD. Targeted detection of murine colonic dysplasia in vivo with flexible multispectral scanning fiber endoscopy. J Biomed Opt 2012;17:021103.

20. Bae SM, Bae DJ, Do EJ, et al. Multi-spectral fluorescence imaging of colon dysplasia invivo using a multi-spectral endoscopy system. Transl Oncol 2019;12:226-235.

21. Yoon SM, Myung SJ, Kim IW, et al. Application of near-infrared fluorescence imaging using a polymeric nanoparticlebased probe for the diagnosis and therapeutic monitoring of colon cancer. Dig Dis Sci 2011;56:3005-3013.

22. Yoon SM, Myung SJ, Ye BD, et al. Near-infrared fluorescence imaging using a protease-specific probe for the detection of colon tumors. Gut Liver 2010;4:488-497.

23. Burggraaf J, Kamerling IM, Gordon PB, et al. Detection of colorectal polyps in humans using an intravenously administered fluorescent peptide targeted against c-Met. Nat Med 2015;21:955-961.

24. Park Y, Ryu YM, Jung Y, et al. Spraying quantum dot conjugates in the colon of live animals enabled rapid and multiplex cancer diagnosis using endoscopy. ACS Nano 2014;8:88968910.

25. Park S, Lim SY, Bae SM, Kim SY, Myung SJ, Kim HJ. Indocyanine-based activatable fluorescence turn-on probe for $\gamma$-glutamyltranspeptidase and its application to the mouse model of colon cancer. ACS Sens 2016;1:579-583.

26. Kim J, Do EJ, Moinova H, et al. Molecular imaging of colorectal tumors by targeting colon cancer secreted protein-2 (CCSP-2). Neoplasia 2017;19:805-816.

27. Bae SM, Park SJ, Choi M, et al. PSP1, a phosphatidylserinerecognizing peptide, is useful for visualizing radiation-induced apoptosis in colorectal cancer in vitro and in vivo. 
Transl Oncol 2018;11:1044-1052.

28. Park Y, Ryu YM, Wang T, et al. Colorectal cancer diagnosis using enzyme-sensitive ratiometric fluorescence dye and antibody-quantum dot conjugates for multiplexed detection. Adv Funct Mater 2018;28:1703450.

29. Yang DH, Rey I. Endoscopic submucosal dissection for colitis-associated dysplasia. Clin Endosc 2019;52:120-128.

30. Jess T, Gamborg M, Matzen P, Munkholm P, Sørensen TI. Increased risk of intestinal cancer in Crohn's disease: a metaanalysis of population-based cohort studies. Am J Gastroenterol 2005;100:2724-2729.

31. Jess T, Rungoe C, Peyrin-Biroulet L. Risk of colorectal cancer in patients with ulcerative colitis: a meta-analysis of population-based cohort studies. Clin Gastroenterol Hepatol 2012; 10:639-645.

32. Kim BJ, Yang SK, Kim JS, et al. Trends of ulcerative colitis-associated colorectal cancer in Korea: a KASID study. J Gastroenterol Hepatol 2009;24:667-671.

33. Mattar MC, Lough D, Pishvaian MJ, Charabaty A. Current management of inflammatory bowel disease and colorectal cancer. Gastrointest Cancer Res 2011;4:53-61.

34. Feagins LA, Souza RF, Spechler SJ. Carcinogenesis in IBD: potential targets for the prevention of colorectal cancer. Nat Rev Gastroenterol Hepatol 2009;6:297-305.

35. Rubin DC, Shaker A, Levin MS. Chronic intestinal inflammation: inflammatory bowel disease and colitis-associated colon cancer. Front Immunol 2012;3:107.

36. Saleh M, Trinchieri G. Innate immune mechanisms of colitis and colitis-associated colorectal cancer. Nat Rev Immunol 2011;11:9-20.

37. Kawanishi S, Hiraku Y, Pinlaor S, Ma N. Oxidative and nitrative DNA damage in animals and patients with inflammatory diseases in relation to inflammation-related carcinogenesis. Biol Chem 2006;387:365-372.

38. van Dieren JM, Wink JC, Vissers KJ, et al. Chromosomal and microsatellite instability of adenocarcinomas and dysplastic lesions (DALM) in ulcerative colitis. Diagn Mol Pathol 2006; 15:216-222.

39. Tahara T, Inoue N, Hisamatsu T, et al. Clinical significance of microsatellite instability in the inflamed mucosa for the prediction of colonic neoplasms in patients with ulcerative colitis. J Gastroenterol Hepatol 2005;20:710-715.

40. Xie J, Itzkowitz SH. Cancer in inflammatory bowel disease. World J Gastroenterol 2008;14:378-389.

41. Itzkowitz S. Colon carcinogenesis in inflammatory bowel disease: applying molecular genetics to clinical practice. J
Clin Gastroenterol 2003;36(5 Suppl):S70-S74.

42. Itzkowitz SH, Harpaz N. Diagnosis and management of dysplasia in patients with inflammatory bowel diseases. Gastroenterology 2004;126:1634-1648.

43. Murthy SK, Kiesslich R. Evolving endoscopic strategies for detection and treatment of neoplastic lesions in inflammatory bowel disease. Gastrointest Endosc 2013;77:351-359.

44. Annese V, Beaugerie L, Egan L, et al. European evidencebased consensus: inflammatory bowel disease and malignancies. J Crohns Colitis 2015;9:945-965.

45. Farraye FA, Odze RD, Eaden J, Itzkowitz SH. AGA technical review on the diagnosis and management of colorectal neoplasia in inflammatory bowel disease. Gastroenterology 2010; 138:746-774.

46. Cairns SR, Scholefield JH, Steele RJ, et al. Guidelines for colorectal cancer screening and surveillance in moderate and high risk groups (update from 2002). Gut 2010;59:666689 .

47. Kornbluth A, Sachar DB; Practice Parameters Committee of the American College of Gastroenterology. Ulcerative colitis practice guidelines in adults: American College of Gastroenterology, Practice Parameters Committee. Am J Gastroenterol 2010;105:501-523.

48. Awais D, Siegel CA, Higgins PD. Modelling dysplasia detection in ulcerative colitis: clinical implications of surveillance intensity. Gut 2009;58:1498-1503.

49. Subramanian V, Ramappa V, Telakis E, et al. Comparison of high definition with standard white light endoscopy for detection of dysplastic lesions during surveillance colonoscopy in patients with colonic inflammatory bowel disease. Inflamm Bowel Dis 2013;19:350-355.

50. Kiesslich R, Neurath MF. Chromoendoscopy in inflammatory bowel disease. Gastroenterol Clin North Am 2012;41:291302.

51. Brown SR, Baraza W, Din S, Riley S. Chromoscopy versus conventional endoscopy for the detection of polyps in the colon and rectum. Cochrane Database Syst Rev 2016;4:CD006439.

52. Watanabe T, Ajioka Y, Mitsuyama K, et al. Comparison of targeted vs random biopsies for surveillance of ulcerative colitis-associated colorectal cancer. Gastroenterology 2016;151: 1122-1130

53. Rubin DT, Rothe JA, Hetzel JT, Cohen RD, Hanauer SB. Are dysplasia and colorectal cancer endoscopically visible in patients with ulcerative colitis? Gastrointest Endosc 2007;65: 998-1004.

54. Rutter MD, Saunders BP, Wilkinson KH, Kamm MA, Williams 
CB, Forbes A. Most dysplasia in ulcerative colitis is visible at colonoscopy. Gastrointest Endosc 2004;60:334-339.

55. American Society for Gastrointestinal Endoscopy Standards of Practice Committee, Shergill AK, Lightdale JR, et al. The role of endoscopy in inflammatory bowel disease. Gastrointest Endosc 2015;81:1101-1121.

56. Laine L, Kaltenbach T, Barkun A, et al. SCENIC international consensus statement on surveillance and management of dysplasia in inflammatory bowel disease. Gastrointest Endosc 2015;81:489-501.

57. Velayos F, Kathpalia P, Finlayson E. Changing paradigms in detection of dysplasia and management of patients with inflammatory bowel disease: is colectomy still necessary? Gastroenterology 2017;152:440-450.

58. Annese V, Daperno M, Rutter MD, et al. European evidence based consensus for endoscopy in inflammatory bowel disease. J Crohns Colitis 2013;7:982-1018.

59. Iacopini F, Saito Y, Yamada M, et al. Curative endoscopic submucosal dissection of large nonpolypoid superficial neoplasms in ulcerative colitis (with videos). Gastrointest Endosc 2015;82:734-738.

60. Suzuki N, Toyonaga T, East JE. Endoscopic submucosal dissection of colitis-related dysplasia. Endoscopy 2017;49:12371242.

61. Yang DH, Kim J, Song EM, et al. Outcomes of ulcerative colitis-associated dysplasia patients referred for potential endoscopic submucosal dissection. J Gastroenterol Hepatol 2019; 34:1581-1589.

62. Kinoshita S, Uraoka T, Nishizawa T, et al. The role of colorectal endoscopic submucosal dissection in patients with ulcerative colitis. Gastrointest Endosc 2018;87:1079-1084.

63. Gounaris E, Martin J, Ishihara Y, et al. Fluorescence endoscopy of cathepsin activity discriminates dysplasia from colitis. Inflamm Bowel Dis 2013;19:1339-1345.

64. Mason SD, Joyce JA. Proteolytic networks in cancer. Trends Cell Biol 2011;21:228-237.

65. Mitsunaga M, Kosaka N, Choyke PL, et al. Fluorescence endoscopic detection of murine colitis-associated colon cancer by topically applied enzymatically rapid-activatable probe. Gut 2013;62:1179-1186.

66. Pompella A, De Tata V, Paolicchi A, Zunino F. Expression of gamma-glutamyltransferase in cancer cells and its significance in drug resistance. Biochem Pharmacol 2006;71:231238.

67. De Palma GD, Colavita I, Zambrano G, et al. Detection of colonic dysplasia in patients with ulcerative colitis using a targeted fluorescent peptide and confocal laser endomicroscopy: a pilot study. PLoS One 2017;12:e0180509.

68. Kiesslich R, Burg J, Vieth M, et al. Confocal laser endoscopy for diagnosing intraepithelial neoplasias and colorectal cancer in vivo. Gastroenterology 2004;127:706-713.

69. Kiesslich R, Goetz M, Lammersdorf K, et al. Chromoscopyguided endomicroscopy increases the diagnostic yield of intraepithelial neoplasia in ulcerative colitis. Gastroenterology 2007;132:874-882.

70. Caobelli F, Panarotto MB, Andreoli F, Ravelli A, De Agostini A, Giubbini R. Is 99mTc-HMPAO granulocyte scan an alternative to endoscopy in pediatric chronic inflammatory bowel disease (IBD)? Eur J Pediatr 2011;170:51-57.

71. Treglia G, Quartuccio N, Sadeghi R, et al. Diagnostic performance of fluorine-18-fluorodeoxyglucose positron emission tomography in patients with chronic inflammatory bowel disease: a systematic review and a meta-analysis. J Crohns Colitis 2013;7:345-354.

72. Caobelli F, Evangelista L, Quartuccio N, et al. Role of molecular imaging in the management of patients affected by inflammatory bowel disease: state-of-the-art. World J Radiol 2016;8:829-845.

73. Wang H, Machtaler S, Bettinger T, et al. Molecular imaging of inflammation in inflammatory bowel disease with a clinically translatable dual-selectin-targeted US contrast agent: comparison with FDG PET/CT in a mouse model. Radiology 2013;267:818-829.

74. Kiesslich R, Duckworth CA, Moussata D, et al. Local barrier dysfunction identified by confocal laser endomicroscopy predicts relapse in inflammatory bowel disease. Gut 2012;61: 1146-1153.

75. Atreya R, Neumann $H$, Neufert $C$, et al. In vivo imaging using fluorescent antibodies to tumor necrosis factor predicts therapeutic response in Crohn's disease. Nat Med 2014;20: 313-318. 